\title{
Why ischemic heart disease and hypertension are nowadays the most serious accident for the affected people?
}

\section{Editorial}

Progresses in the prevention and treatment of cardiovascular disease are continuously observed with promising results. ${ }^{1-3}$ Despite of that, ischemic heart disease and hypertension have to be still interpreted as a serious accident for those individuals who are suffering.

Why that?

Three main factors are related to the outcome of these events:

1. The close relationship between ischemic heart disease, hypertension and major cardiovascular risk factors.

2. The great difficult and complex to follow the therapeutic protocols to prevent/reduce the harmful effects due to these diseases in the patients usually unable to drug assumption.

3. The strong severity, which characterizes the disease itself.

There is evidence that the most dangerous risk factors are often associated with both ischemic heart disease and hypertension, being the latter able to influence the appearance of myocardial damage and its complications. ${ }^{4,5}$ In addition, a damaging factor completely avoidable like cigarette smoking is far to be given up. Thus, consistent data undoubtedly demonstrated a clear defined and reproducible harm to the heart and blood vessel as a result of smoking and the amount of the damage varied according to the type of smoking, its duration and individual exposure and health status of the individuals who met tobacco toxics. High LDL-Cholesterol levels, often associated with metabolic syndrome, adversely influence coronary and carotid arteries being the precursor to atherosclerotic plaque formation and its complications. ${ }^{6,7}$ Oxidized LDL-Cholesterol enters various stages of those processes which lead monocytes to bind endothelial cells and, therefore, the successive migration of them into the intima of the arterial wall. This step contributes to form foam cells within the fatty streaks with secretory and pro-inflammatory process activation. This is the basis of atherosclerosis beginning. Evidence indicates that a large number of livings display LDL-Cholesterol levels at risk.

The progress of cardiovascular therapy is undoubtedly a factor to be positively considered. ${ }^{9}$ However, hypertension and ischemic heart disease often require complex and expensive treatments, which do not meet a high compliance ${ }^{10}$ of the patients.

A metanalysis study ${ }^{11}$ describes a substantial proportion of people do not adhere adequately to cardiovascular medications. Statistically, absolute and relative risk assessments demonstrated that a considerable proportion of all cardiac events ( $~ 9 \%$ in Europe) could be attributed to poor adherence to vascular medications alone, and that the level of optimal adherence gave a significant inverse association with subsequent adverse outcomes. Therefore, measures to enhance adherence to help maximize the potentials of effective cardiac therapies in the clinical setting should be urgently improved.

Finally, the pathological lesions which can accompany both
Volume II Issue 2 - 2018

\author{
Aurelio Leone ${ }^{1,2,3}$ \\ 'Fellow of the American Society of Hypertension (FASH), USA \\ ${ }^{2}$ Fellow of the Royal Society for Promotion of Health (FRSPH), \\ UK \\ ${ }^{3}$ Editor-in-Chief of the Journal of Cardiology \& Current \\ Research, USA
}

Correspondence: Aurelio Leone, Fellow of the American Society of Hypertension (USA), Fellow of the Royal Society for Promotion of Health (UK), Editor-in-Chief of the Journal of Cardiology \& Current Research (USA),Via Provinciale 27, 19030 Castelnuovo Magra, Italy, Email reliol@libero.it

Received: March 29, 2018 | Published: April 16, 2018

myocardium and artery vessels of the hypertensive individuals and individuals suffering from ischemic heart disease clearly show the potential danger experienced.

A wide spectrum of alterations either of degenerative or inflammatory type, which are often associated, explain the substantial reason of death danger in the affected individuals. ${ }^{12,13}$ Three types of necrosis characterize myocardial infarction, which is the most dramatic event of the ischemic heart disease: coagulation necrosis, typically due to a coronarogenic mechanism, coagulative myocytolysis with formation of contract bands as an effect of sympathetic nervous system and adrenergic stimulation, and colliquative myocytolysis, characterized by myocardial fiber lysis, which is a close result of hydrolytic enzyme activity deriving from the material reaching the infarct area. Therefore, it is worth noting that these multiple pathogenic mechanisms able to trigger life-threatening arrhythmias and sudden death are a potential risk factor associated with the ischemic heart disease. In addition, cerebral and renal flow impairment due to vascular stenosis of different degree and extent in several artery beds adversely influence the prognosis of individuals with high blood pressure, primarily in case of malignant hypertension associated with other major cardiovascular risk factors.

\section{Conclusion}

As can be seen, dramatically harmful factors are the pathogenic and pathological mechanism explaining the serious events that people with severe hypertension and ischemic heart disease can suddenly meet with sometimes mortal consequences.

\section{References}

1. Ross G. Strategy of prevention: lessons from cardiovascular disease. $\mathrm{Br}$ Med J. 1981;282(6279):1847-1851.

2. Leone A. Preventive measures to fight cardiovascular disease: Current results and future perspectives. J Cardiol Curr Res. 2015;3(4):00110. DOI: 10.15406/jccr.2015.03.00110. 
3. Hidemasa Oh, Hiroshi I, Shunii S. Challenges to success in heart failure: Cardiac cell therapies in patients with heart diseases. J Cardiol. 2016;68(5):361-367.

4. Leone A. Cardiovascular damage from smoking: A fact or belief? Int $J$ Cardiol. 1993;38(2):113-117.

5. Leone A. Relationship between cigarette smoking and other coronary risk factors for atherosclerosis: Risk of cardiovascular disease and preventive measures. Curr Pharm Des. 2003;9(29):2417-2423.

6. Barter P, Gotto AM, LaRosa JC, et al. HDL cholesterol, very low levels of LDL cholesterol and cardiovascular events. $N$ Engl J Med. 2007;357(13):1301-1310.

7. Kendall DM, Sobel BE, Coulston AM, et al. The insulin resistance syndrome and coronary artery disease. Coron Arter Dis. 2003;14(4):335-348.

8. Assmann G, Schulte H. Relation of high-density lipoprotein cholesterol and triglycerides to incidence of atherosclerotic coronary artery disease (the PROCAM experience. Am J Cardiol. 1992;70(7):733-737.
9. Bonow RO, Carabello BA, Kanu C, et al. ACC/AHA 2006 guidelines for the management of patients with valvular heart disease: a report of the American College of Cardiology/American Heart Association Task Force on Practice Guidelines (writing committee to revise the 1998 Guidelines for the Management of Patients With Valvular Heart Disease): developed in collaboration with the Society of Cardiovascular Anesthesiologists: endorsed by the Society for Cardiovascular Angiography and Interventions and the Society of Thoracic Surgeons. Circulation. 114(5):e84-231.

10. Salvetti A, Versari D. Control of blood pressure in the community: an unsolved problem. Curr Pharm Des. 2003;9(29):2375-2384.

11. Chowdhury R, KhanH, Heydon E, et al. Adherence to cardiovascular therapy: a meta-analysis of prevalence and clinical consequences. Eur Heart J. 2013;34(38):2940-2948.

12. Leone A. Myocardial Infarction. Pathological Relevance and Relationship with Coronary Risk Factors. Curr Pharm Des. 2017;23(22):3205-3216.

13. Baroldi G, Radice F, Schmid G, et al. Morphology of acute myocardial infarction in relation to coronary thrombosis. Am Heart J. 1974;87(1):65-75. 